\begin{tabular}{|c|l|}
\hline Title & Resistivity, carrier concentration, and carrier mobility of electrochemically deposited CdT e films \\
\hline Author(s) & Takahashi, Makoto; U osaki, Kohei; Kita, Hideaki; Y amaguchi, Shoji \\
\hline Citation & $\begin{array}{l}\text { Journal of A pplied Physics, 60(6), 2046-2049 } \\
\text { https://doi.org/10.1063/1.337207 }\end{array}$ \\
\hline Issue Date & 1986-09-15 \\
\hline Doc URL & http://hdl.handle.net/2115/20576 \\
\hline Rights & Copyright $\odot 1986$ A merican Institute of Physics \\
\hline Type & article \\
\hline File Information & A IP60-6.pdf \\
\hline
\end{tabular}

Instructions for use 


\title{
Resistivity, carrier concentration, and carrier mobility of electrochemically deposited ColTe milms
}

\author{
Makoto Takahashi, Kohei Uosaki, ${ }^{a)}$ and Hideaki Kita \\ Department of Chemistry, Faculty of Science, Hokkaido University, Sapporo 060, Japan \\ Shoji Yamaguchi \\ Advanced Materials Laboratory, Mitsubishi Petrochemical Co. Ltd., Yokkaichi 510, Japan
}

(Received 7 March 1986; accepted for publication 5 June 1986)

\begin{abstract}
The electrical type, resistivity, and donor or acceptor concentration of CdTe films deposited electrochemically at various potentials were measured. The carrier mobilities of the films were determined from these results. The deposition potential dependence of the mobility was small and the deposition potential dependence of the resistivity was mainly controlled by the deposition potential dependence of the donor or acceptor concentration. The carrier mobilities were very small compared with those in single crystals due to the scattering of the carriers at grain boundaries.
\end{abstract}

\section{INTRODUCTION}

$\mathrm{CdTe}$ is one of the most important materials for thinfilm solar cell application. ${ }^{1}$ The electrochemical deposition is an attractive method to prepare CdTe films because the physical properties of the films can be easily controlled by the deposition conditions, e.g., deposition potential and bath composition. ${ }^{2}$ To establish the preparation method, it is essential to understand the effect of deposition and post-treatment conditions on the physical properties of CdTe films. $\mathrm{We}^{3-6}$ and others ${ }^{7-9}$ have already reported the effects of the deposition and post-treatment conditions on the composition, electric resistance, and photoelectrochemical properties of CdTe films. However, the detail of the electronic properties, e.g., carrier concentration and carrier mobility, of CdTe films and the effect of deposition conditions on these should be investigated further.

In this paper, we measured the electrical type, resistivity, and donor or acceptor concentration of CdTe films deposited electrochemically at various potentials. The carrier mobilities of the films were determined from these results. The deposition potential dependence of the carrier mobilities was small and the deposition potential dependence of the resistivity was mainly controlled by that of the donor or acceptor concentration. The carrier mobilities were very small compared with those in single crystals due to the scattering of the carriers at grain boundaries.

\section{RI. EXPERIMENT}

CdTe films were deposited cathodically on $\mathrm{Ti}$ sheets (The Japan Lamp Industrial Co. Ltd., $0.2 \mathrm{~mm}$ thick), which were degreased by a chloroform and an ethanol vapor, treated by a $10 \% \mathrm{HF}$ solution to remove an oxide layer and washed by deionized water before use, from an aqueous sulfuric acid solution containing $1 \mathrm{M} \mathrm{CdSO}_{4}$ and $1 \mathrm{mM} \mathrm{TeO}_{2}{ }^{3}$ A potentiostat (Wenking Model 68 FRO.5) was used to control the electrode potential. The reagent grade $\mathrm{CdSO}_{4}$ (purity $99.5 \%$ ) and $\mathrm{TeO}_{2}$ (purity $99 \%$ ) were used as purchased. Water was purified by the Milli $Q$ water purification

\footnotetext{
"Author to whom correspondence should be addressed.
}

system. A usual three-electrode cell was used for the deposition of CdTe films. A platinum sheet and a $\mathrm{Ag} / \mathrm{AgCl}$ electrode were used as a counter and a reference electrode, respectively. The deposition of the films was carried out at room temperature after the electrolyte solutions were deaerated by passing a purified He gas through the solution for about $20 \mathrm{~min}$.

To avoid the complication due to conduction through $\mathrm{Ti}$ substrate, CdTe film was fixed on a glass plate by using epoxy resin, and then $T i$ substrate was removed. ${ }^{10}$ Resistivity of the films was measured in dark at room temperature by means of a four-point probe technique using a specially designed probe (Mitsubishi Petrochemcial Co. Ltd., MCPprobe). To carry out impedance measurements, Au and In were vacuum evaporated onto each end of the films. While Au forms an ohmic contact and In forms the Schottky barrier at $p-\mathrm{CdTe},{ }^{11} \mathrm{Au}$ and In give the Schottky barrier and an ohmic contact, respectively, at $n$-CdTe. ${ }^{12}$ Impedance between the two metal contacts was measured for over a wide range of frequencies $(10 \mathrm{kHz}-100 \mathrm{~Hz})$, by using a potentiostat (Nikko Keisoku NPGS-30ls) and a frequency response analyzer (NF Electronics Co. Ltd., s-5720B), which was controlled by a personal computer (NEC Co. I-td., PC8801 ) via GP-IB interface. The electrical type of the films was determined both by thermoelectric power measurements and by the impedance measurements.

\section{RESULTS}

The dependence of the resistivity on the deposition potential is shown in Fig. 1. The electrical type of the films determined by thermoelectric power measurements is also shown in Fig. 1. These results are essentially in good agreement with the results already reported. ${ }^{4}$

The donor or acceptor concentration of films was obtained by analyzing the results of the impedance measurements. Typical plots of real part versus imaginary part of impedance for a $p$-CdTe film deposited at $-0.35 \mathrm{~V}$ vs $\mathrm{Ag} /$ $\mathrm{AgCl}$ are shown in Fig. 2. Half circles with different radius which depended upon the bias voltage between the film and the barrier metal were obtained. These results were analyzed 


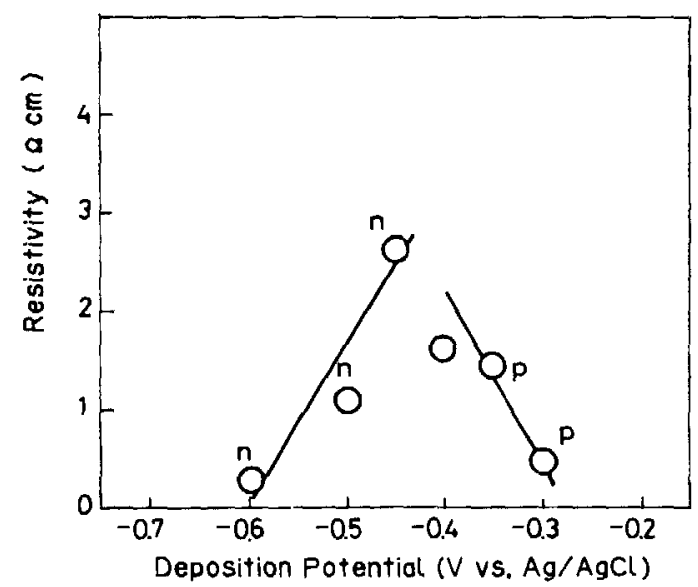

FIG. 1. The electrical type and resistivity of CdTe films as a function of deposition potential.

by using the equivalent circuit also shown in Fig. 2, where $R_{b}, R_{\mathrm{ct}}$, and $C_{\mathrm{sc}}$ are a semiconductor bulk resistance, a charge-transfer resistance, and a space-charge layer capacitance, respectively. While $R_{\mathrm{ct}}$ depended on the applied bias and decreased with the increase of the applied reverse bias, $R_{b}$ was independent of the applied reverse bias and was in good agreement with the value determined by the four-point probe method. The relation between the space-charge capacitance and the reverse bias voltage $V_{\mathrm{bi}}$ is given by

$$
\frac{1}{C_{\mathrm{sc}}^{2}}= \pm \frac{2}{e \epsilon \epsilon_{\mathrm{o}} N}\left(V_{\mathrm{bi}}-\phi_{b}-\frac{k T}{e}\right) \text {, }
$$

where $N$ is the concentration of donor or acceptor which is completely ionized and $\phi_{b}$ is the barrier height between a semiconductor and a metal which forms the Schottky barrier at the semiconductor/metal interface. The positive sign stands for $n$-type and negative sign for $p$-type semiconductors. Thus, the electrical type of semiconductor and donor or acceptor concentration can be determined by plotting $1 / C_{\mathrm{sc}}^{2}$ against bias voltage (Mott-Schottky plot). The MottSchottky plots of films deposited at (a) $-0.35 \mathrm{~V}$ and (b) $-0.45 \mathrm{~V}$ vs $\mathrm{Ag} / \mathrm{AgCl}$ are shown in Fig. 3. In both cases, linear relations between $1 / C_{s c}^{2}$ and $V_{b i}$ were obtained and the electrical type of the films determined from these results

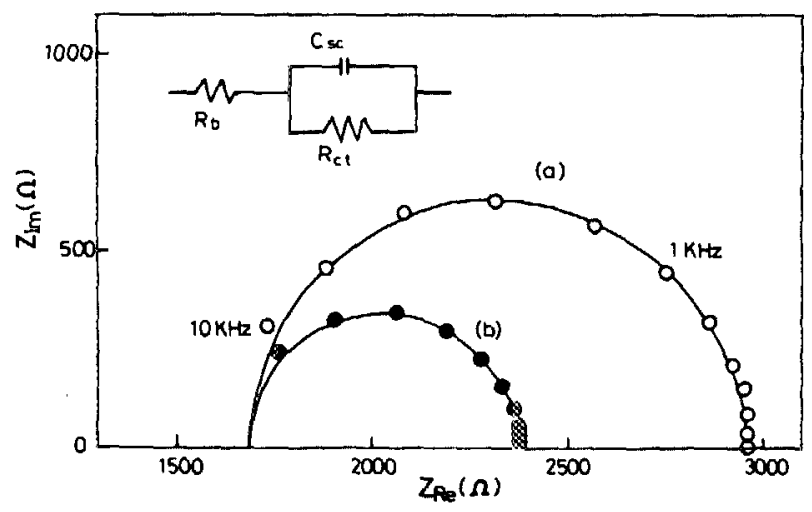

FIG. 2. Imaginary part of impedance vs real part of impedance plots for In/ $p-\mathrm{CdTe}$ film junction. Reverse bias voltage: (a) $0 \mathrm{~V}$, (b) $-0.30 \mathrm{~V}$. The $\mathrm{CdTe}$ film was electrochemically deposited at $-0.35 \mathrm{~V}$ vs $\mathrm{Ag} / \mathrm{AgCl}$. An equivalent circuit for this system is also shown in the figure.

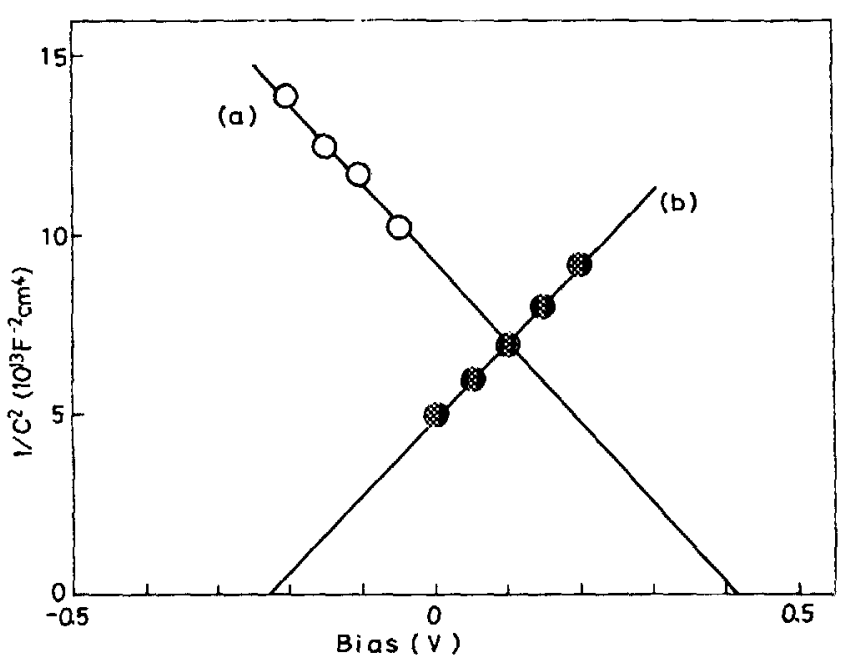

FIG. 3. $C^{-2}$ vs bias voltage plots for (a) In/p-CdTe and (b) Au/ $n-\mathrm{CdTe}$ junctions. $p-\mathrm{CdTe}$ film and $n-\mathrm{CdTe}$ film were deposited at $-0.35 \mathrm{~V}$ and $-0.45 \mathrm{~V}$ vs $\mathrm{Ag} / \mathrm{AgCl}$, respectively.

agreed with those determined by the thermoelectric power measurement. The donor or acceptor concentration determined from the slope and the Schottky barrier heights obtained at the intercept of bias axis are listed in Table I for CdTe films deposited at various potentials.

The barrier heights at $n-\mathrm{CdTe}$ film/Au and at $p$-CdTe/ In interfaces are relatively low compared with those at corresponding single-crystal $\mathrm{CdTe} /$ metal interfaces, ${ }^{13,14}$ suggesting the existence of surface states with a high density.

The donor or acceptor concentration of films is also shown in Fig. 4 as a function of deposition potential. In $p$ type region, the more negative the deposition potential was, the lower the acceptor concentration was. On the other hand, the more negative the deposition potential was, the higher the donor concentration was in $n$-type region.

\section{DISCUSSION}

The potential dependence of the electrical type (Fig. 1) and the donor and acceptor concentration (Fig. 4) can be analyzed by considering that of $\mathrm{Cd} / \mathrm{Te}$ ratio in the $\mathrm{CdTe}$ films. We have already reported that the more negative the deposition potential is, the higher the $\mathrm{Cd} / \mathrm{Te}$ ratio in the electrochemically deposited CdTe films. ${ }^{3.4}$ is known that cadmium vacancy and interstitial tellurium acted as acceptors, while interstitial cadmium and tellurium vacancy acted as donors. ${ }^{15}$ Thus, the more positive the deposition potential is, the more $\mathrm{Te}$ is contained and, therefore, the higher the acceptor concentration is. Similarly, the more negative the deposition potential is, the more $\mathrm{Cd}$ is contained and, therefore, the higher the donor concentration is. The CdTe films deposited at relatively negative potentials which contain less Te are $n$ type and those deposited at relatively positive potentials are $p$ type. The transition of $p$ type to $n$ type occurs at $\sim$ $-0.4 \mathrm{~V}$.

The resistivity $\rho$ is given by

$\rho=1 /\left[e\left(\mu_{h} \mathrm{p}+\mu_{e} n\right)\right]$,

where $n$ is the density of free electrons, $p$ is the density of free 


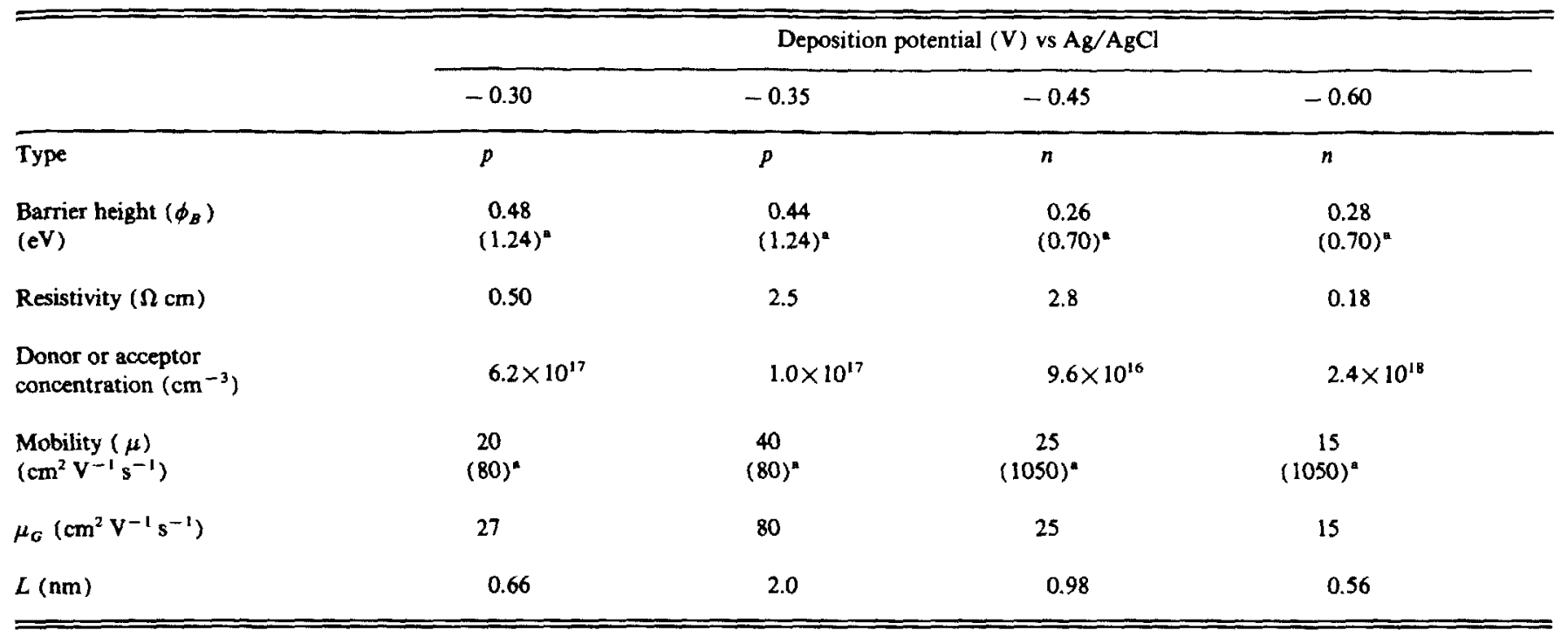

- Values in parentheses are of single crystals.

holes, $\mu_{\mathrm{e}}$ is the electron mobility, and $\mu_{h}$ is the hole mobility. Knowing $\rho, n$, and $p$, carrier mobility can be calculated by using Eq. (2). In the $p$-type region, $p>n$ and, therefore Eq.

(2) can be simplified as

$$
\rho=1 / e p \mu_{h} \text {. }
$$

Similarly, in the $n$-type region where $n>p$,

$$
\rho=1 / e n \mu_{e} \text {. }
$$

It is reasonable to assume the donor concentration in $n$-type region determined by Eq. (1) is equal to density of free electrons. ${ }^{16}$ Similarly, the acceptor concentration in the $p$-type region is equal to density of free holes. Thus, the carrier mobilities can be determined either by using Eq. (3) or (4) with the values of resistivity and the acceptor or donor concentration determined experimentally and are listed also in Table I.

The deposition potential dependence of the mobility is very small and, therefore, the deposition potential dependence of resistivity is mainly controlled by the dependence of the carrier concentration on the deposition potential. In the

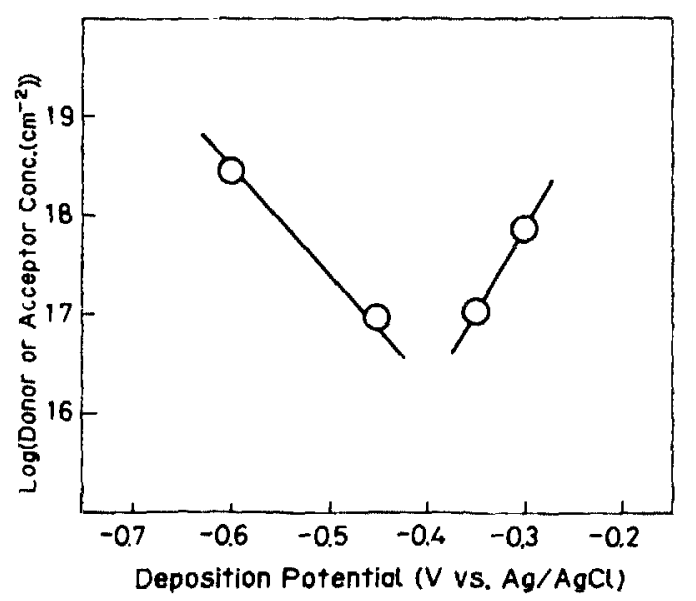

FIG. 4. Potential dependence of donor or acceptor concentration of electrochemically deposited CdTe films. $n$-type region, the more negative the deposition potential, the lower the hole concentration $p$, and therefore, the higher the resistivity [Eq. (3)]. Similarly, in the $n$-type region, the more negative the deposition potential, the higher the electron concentration $n$, and, therefore, the lower the resistivity [Eq. (4)]. At the transition region ( $E_{\text {dep }} \sim-0.40 \mathrm{~V}$ ), both $p$ and $n$ are very small and, therefore, very high resistivity is expected.

The mobilities of both hole and electron of deposited CdTe films were much smaller than those reported for single-crystal CdTe. ${ }^{17}$ The major reason for these small values could be the scattering of carriers at grain boundaries, since the grain size in the CdTe films was very small $(\sim 10 \mathrm{~nm})$. The mobility in polycrystalline solid can be expressed as follows:

$$
\frac{1}{\mu}=\frac{1}{\mu_{L}}+\frac{1}{\mu_{I}}+\frac{1}{\mu_{N}}+\frac{1}{\mu_{\mathrm{G}}},
$$

where $\mu_{L}$ is the lattice limited mobility, $\mu_{I}$ the ionized impurity limited mobility, and $\mu_{G}$ the grain-boundary limited mobility. Similarly, the mobility in a single crystal $\mu_{s}$ is given by

$$
\frac{1}{\mu_{s}}=\frac{1}{\mu_{L}}+\frac{1}{\mu_{t}}+\frac{1}{\mu_{N}} \text {. }
$$

If one assumes that $\mu_{L}, \mu_{I}$, and $\mu_{N}$ have same values both in a polycrystalline and in a single crystal, Eq. (5) can be rewritten as ${ }^{18}$

$$
\frac{1}{\mu}=\frac{1}{\mu_{s}}+\frac{1}{\mu_{G}} \text {. }
$$

Thus, $\mu_{G}$ can be calculated by using the values of $\mu_{s}$ reported for a single crystal and $\mu$ determined experimentally. The values of $\mu_{G}$ thus obtained for the CdTe films are also listed in Table $\mathrm{I}$. It is clearly shown that the mobility in the CdTe films are almost solely controlled by the grain boundary limited mobility. $\mu_{G}$ is related to the mean free path of the carrier as

$$
\mu_{G}=e \tau_{G} / m^{*},
$$


where $m^{*}$ is the reduced mass of the carrier and $\tau_{G}$ is the grain-boundary controlled relaxation time which is given by

$$
\tau_{G}=L / v
$$

In the above equation, $L$ is the grain-boundary limited mean free path and $v$ is the average thermal velocity given by

$$
v=\left(3 k T / m^{*}\right)^{1 / 2} .
$$

By using Eqs. (8) and (9), $T=300 \mathrm{~K}, m_{e}^{*} / m=0.14,{ }^{17}$ $m_{h}^{*} / m=0.35,{ }^{17}$ and the values of $\mu_{G}$, the values of $L$ are calculated and are summarized also in Table $I$. The calculated values of $L$ are very sma11 $(0.5-2 \mathrm{~nm})$ and are similar to those reported for polycrystalline InP, the grain size of which is $\sim 15 \mu \mathrm{m} .^{18}$ These results show that the mobilities of carriers in the electrodeposited CdTe films are limited mainly by grain boundary scattering as expected. $\mu$ as well as $\mu_{G}$ and $L$ should increase with the increase of the grain size. Actually, our preliminary results showed that $\mu, \mu_{G}$, and $L$ increased by heat treatment in $\mathrm{He}$ atmosphere which increased grain size.

\section{ACXNOWLEDGNENT}

This work is partially supported by a Grant-in-Aid for
Scientific Research from Ministry of Education, Science and Culture.

'A. Rothwarf and K. W. Boer, Prog. Solid-State Chem. 10, 71 (1975). ${ }^{2}$ M. P. R. Panicker, M. Knaster, and F. A. Kroger, J. Electrochem. Soc. 125, 566 (1978).

${ }^{3}$ M. Takahashi, K. Uosaki, and H. Kita, J. Electrochem. Soc. 131, 2304 (1984).

${ }^{4}$ M. Takahashi, K. Uosaki, and H. Kita, J. Appl. Phys. 55, 3879 (1984).

${ }^{5}$ M. Takahashi, K. Uosaki, and H. Kita, J. Appl. Phys. 58, 4292 (1985).

'M. Takahashi, K. Uosaki, and H. Kita, J. Electrochem. Soc. 133, 258 (1986).

7J. Llabres, J. Electrochem. Soc. 131, 468 (1984).

${ }^{8}$ B. M. Basol and O. M. Stafsudd, Thin Solid Films 78, 217 (1981).

${ }^{9}$ B. M. Basol and O. M. Stafsudd, Solid-State Electron. 24, 121 (1981).

${ }^{10}$ A. B. Baranski, M. S. Bennett, and W. R. Fawcett, J. Appl. Phys. 54, 6390 (1983).

"J. G. Werthen, J. P. Häring, A. L. Fahrenbruch, and R. H. Bube, J. Appl. Phys. 54, 5982 (1983).

${ }^{12}$ G. Fulop, M. Doty, P. Meyers, J. Betz, and C. H. Liu, Appl. Phys. Lett. 40, 327 (1982).

${ }^{13}$ I. M. Dharmadasa, G. G. Roberts, and M. C. Petty, J. Phys. D. 15, 901 (1982).

${ }^{14}$ N. Nishimura and R. H. Bube, J. Appl. Phys. 58, 420 (1985).

${ }^{15}$ J. Saraie, M. Kitagawa and T. Tanaka, J. Electrochem. Soc. 126, 2226 (1979)

${ }^{16} \mathrm{~V}$. A. Myamlin and Yu. V. Pleskov, Electrochemistry of Semiconductors, English Edition (Plenum, New York, 1967), p. 10.

${ }^{17}$ D. de Noble, Philips Res. Rep. 14, 487 (1959).

${ }^{18}$ J. N. Roy, S. Basu, and D. N. Bose, J. Appl. Phys. 54, 847 (1983). 
Journal of Applied Physics is copyrighted by the American Institute of Physics (AIP). Redistribution of journal material is subject to the AIP online journal license and/or AIP copyright. For more information, see http://ojps.aip.org/japo/japcr/jsp

Copyright of Journal of Applied Physics is the property of American Institute of Physics and its content may not be copied or emailed to multiple sites or posted to a listserv without the copyright holder's express written permission. However, users may print, download, or email articles for individual use. 